\title{
Traducción al español de la escala de adicción a los alimentos de Yale (Yale Food Addiction Scale) y su evaluación en una muestra de población mexicana. Análisis factorial
}

\author{
Mariana Isabel Valdés Moreno, ${ }^{1,2}$ María Cristina Rodríguez Márquez, ${ }^{2}$ Juan José Cervantes Navarrete, ${ }^{3}$ \\ Beatriz Camarena, ${ }^{4}$ Patricia de Gortari ${ }^{1}$
}

Artículo original

\section{ABSTRACT}

\section{Introduction}

Obesity has a multifactorial etiology and is a global public health problem which also affects Mexican population. Obesity is characterized by excessive body adiposity, as well as high prevalence of diverse comorbidities, which diminish life quality. Sedentary lifestyle and hypercaloric food overconsumption are amongst the causes of obesity. It has been suggested that some traits seen in obese patients may represent an addictive behavior, similar to those observed in substance-dependent patients.

\section{Objective}

The aim of this work was the validation of the Spanish version of the Yale Food Addiction Rating Scale (YFAS) in a Mexican adult population sample.

\section{Method}

The scale was applied twice to 160 participants with a three weeks period in-between. The factorial model was corroborated with Bartlett's sphericity test and with that of Kaiser-Meyer-Olkin.

\section{Results}

Internal consistency was calculated by means of Cronbach's alpha which was $\alpha=0.7963$; reliability, measured with Spearman's coefficient by means of the test-retest method, was $r=0.565, n=96$. Convergence validity was estimated using the Binge Eating Scale (BES) and Spearman's correlation $(r=0.5868 p \leq 0.0001 ; n=157)$. Bartlett's sphericity test showed $\left(\chi^{2}{ }_{(300)}=1572.33, p<0.05\right)$ and that of Kaiser-Meyer-Olkin (KMO $=0.825)$, showing consistency for the factorial model. Spearman's correlation between YFAS and body mass index (BMI) showed $r=0.2843 p \leq 0.001 ; n=151$.

\section{Discussion and conclusion}

The Spanish version of the YFAS showed psychometric properties not different from the original and adapted existing versions. Therefore, YFAS Spanish version could be useful in healthcare and clinical research in Mexican population.

Key words: Obesity, addictive behavior, validation study.

\section{RESUMEN}

\section{Introducción}

La obesidad es la acumulación excesiva de grasa corporal, lo que condiciona una alta comorbilidad. El consumo descontrolado de alimentos hipercalóricos es causa de su desarrollo; ésta es una conducta de características similares a la de pacientes con adicción a sustancias. La escala de adicción a los alimentos, YFAS (Yale Food Addiction Scale), permite identificar a sujetos con conducta adictiva a los alimentos.

\section{Objetivo}

Validar la escala YFAS en español en una muestra de población mexicana adulta.

\section{Método}

La muestra de participantes (160) respondió a la encuesta en dos ocasiones, con un período de tres semanas entre cada aplicación. La pertinencia de un modelo factorial se corroboró con las pruebas de esfericidad de Bartlett y la medición del parámetro de Kaiser-Meyer-Olkin.

\section{Resultados}

El $\alpha$ de Cronbach $=0.7963$ corroboró la consistencia interna de la escala. Para la confiabilidad se obtuvo el coeficiente de Spearman

Laboratorio de Neurofisiología Molecular, Instituto Nacional de Psiquiatría Ramón de la Fuente Muñiz.

Escuela de Dietética y Nutrición del ISSSTE.

Subdirección de Hospitalización, Instituto Nacional de Psiquiatría Ramón de la Fuente Muñiz.

Departamento de Farmacogenética, Instituto Nacional de Psiquiatría Ramón de la Fuente Muñiz.

Correspondencia: Dra. Patricia de Gortari, Laboratorio de Neurofisiología Molecular, Dirección de Investigaciones en Neurociencias, Instituto Nacional de Psiquiatría Ramón de la Fuente Muñiz, Calzada México-Xochimilco 101, Col. San Lorenzo Huipulco, Tlalpan, C.P. 14370, Ciudad de México, México. Tel: 4160 - 5056. E-mail: gortari@imp.edu.mx

Recibido primera versión: 10 de febrero de 2016. Segunda versión: 23 de agosto de 2016. Aceptado: 24 de octubre de 2016 
por la metodología test-retest, de $r=0.565, n=96$. La validación por convergencia, correlacionando con la escala para trastorno por atracón (Binge Eating Scale, BES) $(r=0.5868 p \leq 0.0001 ; n=157)$. Las pruebas de Bartlett $\left(\chi_{(300)}^{2}=1572.3, p<0.05\right)$ y Kaiser-Meyer-Olkin $(K M O=0.825)$ mostraron pertinencia del modelo factorial. Además, se correlacionaron los resultados de la YFAS con el índice de masa corporal (IMC) $(r=0.2843 p \leq 0.001 ; n=151)$.

\section{Discusión y conclusión}

Esta versión de YFAS presentó propiedades psicométricas adecuadas y similares a las de la original y a otras de sus traducciones y adaptaciones. Se considera entonces con utilidad para la práctica asistencial y para estudios de investigación clínica en población mexicana.

Palabras clave: Obesidad, adicción a los alimentos, validez.

\section{INTRODUCCIÓN}

El consumo desmedido de alimentos es un fenómeno que recientemente ha cobrado interés, sobre todo por ser una de las causas de la obesidad, una pandemia asociada a comorbilidades potencialmente fatales, ${ }^{1}$ que, a pesar del progreso médico y de las acciones gubernamentales, no ha sido posible revertir. ${ }^{2}$

La obesidad es una acumulación excesiva de grasa corporal que representa riesgos para la salud y se define por un IMC mayor o igual a $30 \mathrm{Kg} / \mathrm{m}^{2}{ }^{2}$. Según la Organización Mundial de la Salud (OMS), la obesidad se ha duplicado desde 1980. En 2014, más de mil novecientos millones de adultos (39\%) tenían un índice de masa corporal (IMC) mayor a $25 \mathrm{Kg} / \mathrm{m}^{2}$, y de éstos, 600 millones (13\%) se encontraban en el rango de obesidad. ${ }^{4}$

El sedentarismo y el consumo desmedido de alimentos hipercalóricos (ricos en azúcar y grasa), aun en presencia de reservas energéticas corporales suficientes, parecen rebasar el control homeostásico innato que acopla la conducta alimentaria con el gasto de energía. ${ }^{5-7}$

Diversos estudios clínicos han demostrado que el trastorno por atracón (TPA) presenta una alta frecuencia en sujetos con obesidad, por lo que se ha propuesto que contribuye al desarrollo de la obesidad. ${ }^{8-12}$ La asociación entre la obesidad y la presencia de atracones se ha relacionado con el grado de adiposidad, rasgos psicopatológicos comórbidos y la respuesta al tratamiento. ${ }^{13-15}$

El TPA se caracteriza por episodios recurrentes de atracones de comida en ausencia de comportamiento regular compensatorio, como el vómito o el abuso de laxantes. Algunas características relacionadas incluyen comer hasta sentirse incómodamente lleno, comer cuando no se tiene hambre, comer solo y con sentimientos depresivos o de culpa. ${ }^{16}$ El TPA se ha asociado con distintas entidades psicopatológicas como el trastorno depresivo mayor, trastornos de ansiedad y trastornos de personalidad, que son también comorbilidades de la obesidad. ${ }^{17,18}$

Aunque el TPA no se limita a personas con obesidad, su asociación es más común en este grupo que con algún otro trastorno de la conducta alimentaria. En la población general, la prevalencia del TPA se ha estimado entre un 2 y $5 \%$, mientras que en los individuos que buscan tratamiento para el control de peso la prevalencia es del $30 \% .{ }^{19}$
El origen del consumo compulsivo de alimentos palatables ha sido homologado con la adicción a las drogas en cuanto a la participación del sistema dopaminérgico de recompensa y como consecuencia de la activación de mecanismos de reforzamiento positivo, en este caso generado por los alimentos de alto contenido calórico. ${ }^{20-22}$

Si bien el concepto de adicción a los alimentos aún no se ha aceptado clínicamente, existen evidencias de paralelismos en la neuroquímica de sujetos adictos a drogas y en sujetos con obesidad, ${ }^{23,24}$ sugiriendo que en algunos casos esta última podría ser el resultado de la "adicción a la comida" y que por lo tanto podría ser evaluada considerando los patrones conductuales y de sintomatología observados en otras adicciones. ${ }^{25}$

Dichos patrones han sido plasmados en la escala de adicción a los alimentos de Yale o YFAS (Yale Food Addiction Scale), cuyo objetivo es operacionalizar el concepto de adicción a los alimentos, traduciendo los criterios diagnósticos para los trastornos relacionados con sustancias señalados en el DSM-5, para su aplicación en la conducta alimentaria. ${ }^{26}$

La versión original de esta escala ha probado tener validez interna (coeficiente de Kuder Richardson $\alpha=0.86$ ) y convergente, obtenida al comparar el instrumento con las escalas de actitudes hacia la comida (Eating Attitudes Test26, EAT-26) y la de emociones hacia los alimentos (Emotional Eating Scale, EES), obteniendo en ambos casos correlaciones significativas. La validación discriminativa ha sido resultado de una comparación con el cuestionario de consumo diario de alcohol (Daily Drinking Questionnaire, DDQ), con el índice de Routgers para problemas de alcohol (Routgers Alcohol Problem Index, RAPI) y como instrumento para evaluar la impulsividad se han empleado las escala de inhibición del comportamiento y la de aproximación del comportamiento basadas en el modelo de Alan Gray conocidas como BIS/BAS reactivity (Gray's Behavorial Inhibition/ Behavioral Approach System). La validez discriminativa se considera adecuada puesto que no hay una correlación significativa con el DDQ ni con el BAS y sólo una pequeña pero significativa correlación con el RAPI y con el BIS. ${ }^{26}$

La YFAS es también un indicador del trastorno por atracón medido con la escala para trastorno por atracón (Binge Eating Scale, BES), que evalúa la entidad clínica de trastorno por atracón, reconocida por el DSM-5 55 y que equivaldría al fenómeno de adicción a los alimentos. ${ }^{26}$ 
La YFAS se ha utilizado en numerosos estudios y las traducciones de sus versiones en alemán, francés y español (aplicado en España) ya han sido validadas obteniendo un $\alpha$ de Cronbach de 0.81 - 0.83, 0.90 y 0.82, respectivamente. ${ }^{27-31}$ Se ha desarrollado también una versión corta de la escala, misma que comprende únicamente nueve ítems, uno para cada aspecto conductual de la adicción (siete ítems) más dos ítems para evaluar manifestaciones clínicas significativas $(\alpha=0.75-0.84) .^{32}$ Así mismo, existe una versión de la escala adaptada para niños $(\alpha=0.78) .{ }^{33}$

Considerando la alta prevalencia del consumo compulsivo de comida reportado por los pacientes con obesidad, ${ }^{34}$ también es importante señalar que la obesidad puede ser resultado de alteraciones metabólicas que llevan a un desequilibrio energético, es decir, a un menor gasto de glucógeno y lípidos a pesar de que sus reservas sean grandes.

La regulación de dichas señales por las hormonas leptina, insulina y grelina, que son liberadas del tejido adiposo, páncreas o estómago, están dadas por el tamaño, volumen de los adipocitos o reservas de glucógeno, así como por la presencia de nutrimentos en el sistema digestivo.

Es posible que la liberación de estas hormonas y su señalización puedan encontrarse alteradas en pacientes con obesidad y, en esas condiciones, su tratamiento estaría enfocado en resolver el problema metabólico. Sin embargo, una alteración psiquiátrica también puede estar involucrada. Los trastornos del estado de ánimo son frecuentes en pacientes con obesidad y éstos pueden provocar un consumo de alimentos descontrolado en función del efecto ansiolítico en que se ha implicado a las grasas e hidratos de carbono.

Así pues, la relevancia de contar con un instrumento como éste, traducido al español y adaptado a los alimentos y términos comúnmente usados en México, representa la posibilidad de detectar en la población mexicana una conducta de hiperfagia que puede reflejar rasgos de trastornos psiquiátricos que lleven al desarrollo de la obesidad.

\section{MÉTODO}

\section{Muestra}

Estudio transversal y descriptivo que empleó un muestreo por conveniencia que incluyó 160 trabajadores y estudiantes de distintas instancias del ISSSTE, como la Escuela y el Centro de Dietética y Nutrición del ISSSTE entre febrero y julio de 2015.

\section{Instrumentos}

La escala de Yale es un instrumento de 25 reactivos que analiza la manifestación de los criterios propuestos por el DSM5 para identificar la dependencia a sustancias. ${ }^{25}$
La escala incluye opciones de puntaje dicotómicas y de frecuencia: las frecuencias (nunca, una vez al mes, 2-4 veces al mes, 2-3 veces a la semana, 4 o más veces a la semana o diario: correspondientes a $0,1,2,3$ o 4 puntos, respectivamente) evalúan conductas que pueden presentarse ocasionalmente en individuos sin problemas de adicción, mientras que las opciones dicotómicas (sí o no: 0 o 1 punto) se usan en preguntas que indican problemas más severos de consumo de alimentos. Finalmente, los valores se analizan para determinar si cada uno de los criterios son cumplidos o no. El diagnóstico de adicción a los alimentos se da si el sujeto cumple con al menos tres criterios positivos. ${ }^{35}$

En nuestro estudio, la adaptación de la YFAS al español se realizó por traducción directa y fue revisada por dos psiquiatras, dos especialistas en neurociencias y dos especialistas en nutrición, todos hispanoparlantes nativos con buen manejo del idioma inglés, con el objetivo de traducir y optimizar la claridad de las preguntas.

La BES es un instrumento autoaplicable desarrollado para identificar TPA en personas con obesidad; mide aspectos conductuales como el consumo de grandes cantidades de alimentos y sentimientos o emociones en torno a un episodio de atracón. Esta herramienta ha probado ser útil para identificar a individuos con TPA y para evaluar la intensidad del trastorno. También permite discriminar entre individuos con TPA moderado o severo e individuos sin este trastorno. ${ }^{36-41}$

\section{Procedimiento}

Se obtuvo el peso y talla de cada participante utilizando una báscula Tanita (TBT-215, Tokio, Japón). Los puntos de corte para definir IMC normal, sobrepeso y obesidad fueron los señalados por la OMS: 18.5 - $24.9 \mathrm{Kg} / \mathrm{m}^{2}$ (normal), 25 - 29.9 $\mathrm{Kg} / \mathrm{m}^{2}$ (sobrepeso) y $\geq 30 \mathrm{Kg} / \mathrm{m}^{2}$ (obesidad). ${ }^{42}$

Los criterios de inclusión considerados para seleccionar a los participantes fueron: hombres y mujeres que acudieran por primera vez al Centro de Dietética y Nutrición de la Escuela de Dietética y Nutrición del ISSSTE, edad entre 18 y 45 años y que aceptaran participar en el estudio mediante la firma de la carta de consentimiento informado.

Los criterios de exclusión fueron: mujeres en período de menopausia, fumadores, pacientes con tratamiento antidepresivo o con fármacos anti-obesidad, pacientes en dietoterapia y aquellos con consumo crónico de alcohol (más de dos bebidas alcohólicas por día), dado que son factores que pueden modificar por ellos mismos la conducta alimentaria.

Los participantes fueron instruidos para responder la escala considerando su alimentación en el último año y, en particular, su consumo de alimentos ricos en grasa y/o azúcar, que son los más consumidos durante los atracones.

Se compararon las frecuencias de participantes por género e IMC mediante la prueba de $\chi^{2}$ considerándose una diferencia significativa cuando el valor de $p<0.05$. 


\section{Análisis estadístico}

La consistencia interna se determinó mediante el cálculo del a de Cronbach que se consideró adecuado cuando fue mayor a 0.7. La validación por convergencia se realizó por correlación de Spearman respecto a la BES considerándose significativa cuando el valor de $p<0.05$. El método empleado para evaluar la confiabilidad de la YFAS, es decir, la estabilidad entre dos mediciones de la misma escala, fue el de test-retest (tres semanas de diferencia entre la primera y segunda aplicación) y el cálculo del coeficiente de Spearman respecto a las dos mediciones, considerándose adecuado cuando $p<0.05$.

\section{Análisis factorial}

Con el objetivo de definir la pertinencia de un modelo factorial se realizaron las pruebas de esfericidad de Bartlett y la medición del parámetro de Kaiser-Meyer-Olkin. Posteriormente, se utilizó el procedimiento de rotación factorial de los componentes bajo el sistema de normalización Varimax. Se utilizó el programa SPSS para este análisis.

Se realizó también una correlación de Spearman entre los puntajes de la escala YFAS y el IMC, que se consideró significativa cuando $p<0.05$. Los análisis estadísticos fueron realizados utilizando el programa GraphPad Prism versión 6.

\section{RESULTADOS}

Se reclutaron 160 participantes entre febrero y julio de 2015 y se excluyeron aquéllos que no regresaron a la segunda aplicación de la escala; o bien, a quienes no contestaron todos los ítems de las dos escalas. La muestra de estudio estuvo conformada por individuos de 37 DE 14 años de los cuales $123(77 \%)$ fueron mujeres $\left(\chi^{2}=153.342, p<0.001\right)$. En cuanto al IMC, 71 (46\%) participantes se ubicaron en el rango normal, 51 (33\%) en el rango de sobrepeso y 31 (21\%) en el rango de obesidad. De la totalidad de sujetos con obesidad, 20 (65\%) tuvieron obesidad grado I, 8 (26\%)

\section{Tabla 1}

Estadísticos de adecuación muestral

Medida de Kaiser-Meyer-Olkin de 0.825 adecuación de muestreo

Prueba de esfericidad de Bartlett Aprox Chi-cuadrado 1572.330 gl. Sig. 300

Nota: En la tabla se presenta la medida de adecuación muestral de Kaiser-Me yer-Olkin (KMO), que indica la proporción de la varianza que tienen en común las variables analizadas. El coeficiente $\mathrm{KMO}$ fue cercano a la unidad (KMO = 0.825 ) apoyando que la adecuación del modelo al análisis factorial es buena. La prueba de esfericidad de Bartlett contrasta la hipótesis nula de que la matriz de correlación es una matriz de identidad, en cuyo caso, no existirían correlaciones significativas entre las variables y el modelo no sería pertinente. Sin embargo, los valores de la prueba corroboran que los datos poseen las características adecuadas para la realización de un análisis factorial. tuvieron obesidad grado II y $3(9.9 \%)$ tuvieron obesidad mórbida $\left(\chi^{2}=300.319, p<0.001\right)$.

Considerando la muestra sin distinción de género, $46.1 \%$ tuvo un IMC normal y 53.9\% fue el porcentaje combinado entre sobrepeso y obesidad $\left(\chi^{2}=3.817, p=0.148\right)$.

\section{Consistencia interna}

El valor del a de Cronbach para los 22 reactivos transformables fue de 0.7963. El coeficiente de Spearman para las dos aplicaciones de la escala de Yale fue $r=0.565, p<0.01, n=96$.

\section{Análisis factorial}

Los resultados de la prueba de esfericidad de Bartlett arrojaron valores que comprobaron la adecuación de la matriz para el análisis, indicando pertinencia del modelo factorial (tabla 1). Usando el método de extracción de componentes principales se identificaron siete componentes con valores propios mayor a la unidad $(7.82,5.88,4.32,3.10,1.35,1.30$, 1.1) que explicaron el $72 \%$ de la varianza muestral.

En la tabla 2 se observan las saturaciones de los reactivos en cada uno de los factores bajo el sistema de normalización Varimax.

De los cinco componentes principales el primero consta de los reactivos 1, 2, 3, 4, 5, 6, 7, 12, 13 y 20, los cuales representan tres de los siete criterios que conforman el constructo de adicción a sustancias y de cuyo modelo fue extraído el mismo concepto. Estos son: consumo de la sustancia en mayor cantidad o durante más tiempo que lo que el sujeto pretende, emplear mayor tiempo en obtener-usar la sustancia, presentar síntomas de abstinencia.

El segundo componente consta de las preguntas $9,15,17,19$ y 21 . Estas corresponden a criterios de tolerancia, es decir, continuación del uso a pesar de conocer sus consecuencias negativas y de los que describen la disfunción y el malestar secundario.

El tercer componente, formado por las preguntas 22, 23, 24 y 25, representa el criterio de deseo continuo o incapacidad para reducir o cesar el consumo.

El cuarto componente estuvo formado por las preguntas 8, 10 y 14 que constituye el criterio de reducción o cese de actividades sociales ocupacionales o recreativas a excepción de la pregunta 14 que es parte del criterio de abstinencia.

El quinto componente se constituye por las preguntas 11, 15 y 18 que se corresponde con las consecuencias de malestar y disfunción y con el criterio de reducción o cese de actividades sociales ocupacionales o recreativas.

Se incluye también la varianza total explicada (tabla 3)

\section{Validez convergente}

El coeficiente de Spearman para los puntajes obtenidos en la escala de Yale y en la de Gormally (BES) fue: $r=0.5868$ $p \leq 0.0001 ; n=157$. 


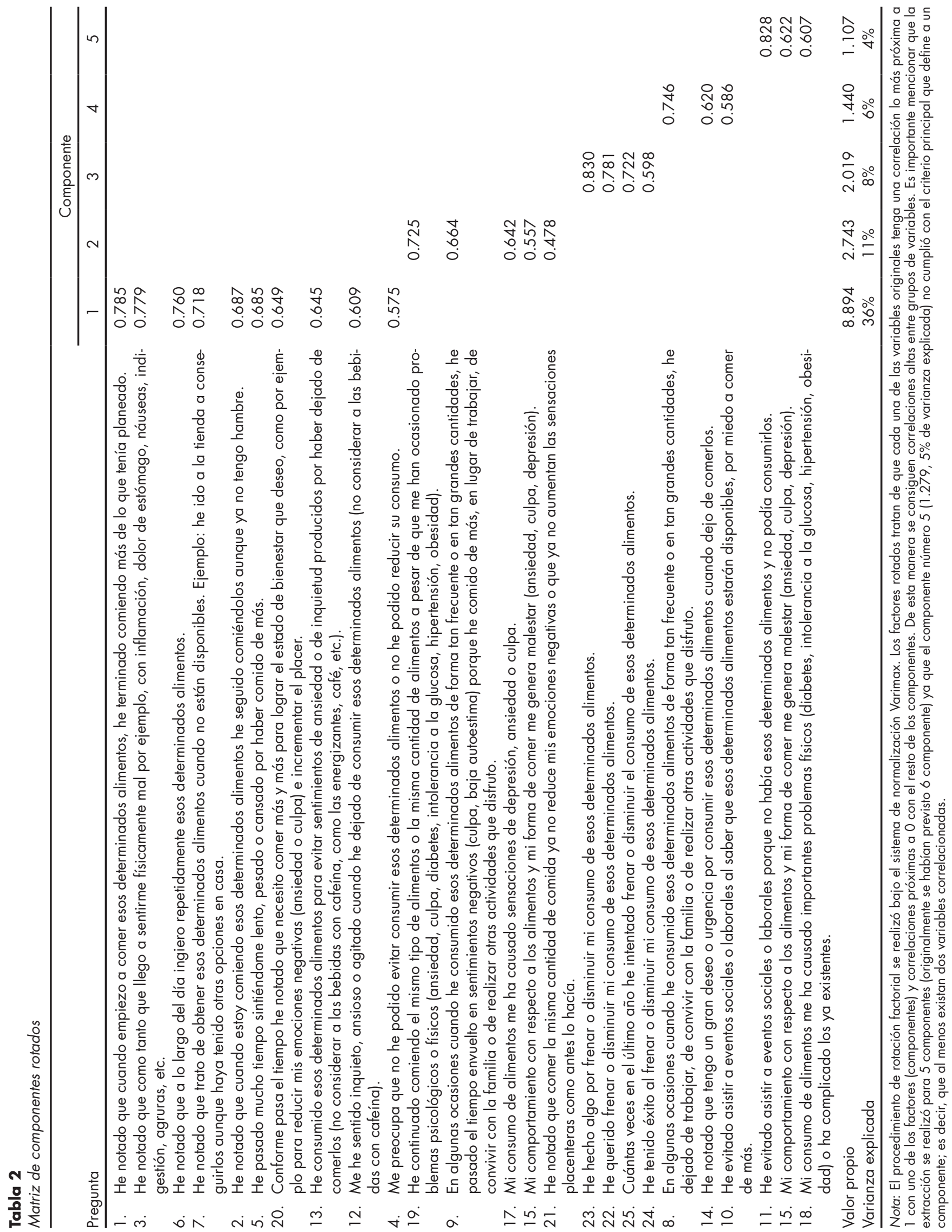


Tabla 3

Varianza total explicada. Los datos muestran las cargas de cada ítem

\begin{tabular}{|c|c|c|c|}
\hline \multirow[b]{2}{*}{ Item } & \multicolumn{3}{|c|}{ Autovalores iniciales } \\
\hline & Total & $\%$ de varianza & $\%$ acumulado \\
\hline 1 & 8.894 & 35.578 & 35.578 \\
\hline 2 & 2.743 & 10.972 & 46.550 \\
\hline 3 & 2.019 & 8.076 & 54.626 \\
\hline 4 & 1.440 & 5.759 & 60.385 \\
\hline 5 & 1.279 & 5.117 & 65.502 \\
\hline 6 & 1.107 & 4.430 & 69.932 \\
\hline 7 & 0.923 & 3.691 & 73.623 \\
\hline 8 & 0.774 & 3.096 & 76.718 \\
\hline 9 & 0.731 & 2.925 & 79.643 \\
\hline 10 & 0.725 & 2.902 & 82.544 \\
\hline 11 & 0.566 & 2.262 & 84.806 \\
\hline 12 & 0.471 & 1.883 & 86.690 \\
\hline 13 & 0.435 & 1.741 & 88.431 \\
\hline 14 & 0.419 & 1.676 & 90.107 \\
\hline 15 & 0.340 & 1.359 & 91.466 \\
\hline 16 & 0.328 & 1.313 & 92.778 \\
\hline 17 & 0.310 & 1.240 & 94.018 \\
\hline 18 & 0.277 & 1.109 & 95.127 \\
\hline 19 & 0.245 & 0.981 & 96.108 \\
\hline 20 & 0.213 & 0.852 & 96.960 \\
\hline 21 & 0.196 & 0.782 & 97.742 \\
\hline 22 & 0.189 & 0.757 & 98.499 \\
\hline 23 & 0.155 & 0.619 & 99.118 \\
\hline 24 & 0.126 & 0.504 & 99.622 \\
\hline 25 & 0.094 & 0.378 & 100.000 \\
\hline
\end{tabular}

Nota: El método de extracción fue el análisis de componentes principales.

\section{Correlación con el IMC}

El coeficiente de Spearman respecto a los puntajes en la escala de Yale y el IMC fue $r=0.2843 p \leq 0.001 ; n=151$.

\section{DISCUSIÓN Y CONCLUSIÓN}

En este trabajo se calculó la consistencia interna y la validez convergente de una versión en español de la YFAS en una muestra de sujetos de la población mexicana.

El valor de la consistencia interna se refirió a la relación entre los ítems de la escala como un todo, es decir, qué tanto de cada ítem midió lo que se deseaba medir con la escala o instrumento. ${ }^{43}$ Diferentes estudios consideran como aceptables, valores de $\alpha$ entre 0.7 y $0.95,,^{44-47}$ lo cual indica que la confiabilidad de nuestra versión de la escala fue adecuada.

La validez convergente obtenida a través del coeficiente de Spearman entre los puntajes obtenidos en los dos instrumentos (YFAS y BES) sugirió una relación lineal positiva entre ambos, lo cual se vincula con los criterios que evalúan ambas escalas, es decir, una conducta alimentaria excesiva episódica, asociada a componentes emocionales como culpa y ansiedad, a pesar de la exacerbación de padecimientos clínicos; por ejemplo, diabetes mellitus tipo 2 e hipertensión.
En resumen, la obtención de un resultado positivo en la YFAS sugiere que el resultado será similar al obtenido utilizando la BES y viceversa.

Además, la BES tiene como propósito medir conductas que son lo más parecido a los criterios diagnósticos sugeridos en la clínica para la "adicción a los alimentos". ${ }^{36}$

En cuanto al análisis factorial, se determinaron cinco componentes que incluyeron todos los reactivos en comparación con el estudio original que determinó cuatro, incluyendo sólo 21 de los 25 reactivos. Las diferencias pudieron deberse a que en el estudio actual los factores dos y cinco conformaron los criterios de las consecuencias de malestar y disfunción, mientras que el original separó aquellas preguntas que examinan ambos.

El primer componente fue el más extenso y el que representa la esencia de constructo de adicción por los alimentos en cuanto a lo que implica un mayor y desmedido consumo. El segundo describió bien las consecuencias asociadas a este consumo desmedido. El tercero expresó adecuadamente lo frágil que resulta la voluntad en cuanto al esfuerzo por frenar el consumo. El cuarto componente expresó el descuido de las demás actividades. Finalmente, el quinto estuvo compuesto en su mayoría por aquellas preguntas que exploran la disfunción y el malestar y que fueron excluidas del estudio original. En general, se conservó la estructura factorial de los criterios que conforman el constructo de adicción a los alimentos.

En cuanto a la correlación entre la escala de Yale y el IMC, el coeficiente de Spearman sugirió una relación positiva débil, lo que implica que podría presentarse una conducta adictiva a los alimentos aun en individuos con peso normal. Sería recomendable un muestreo que considerara una población con mayor prevalencia de obesidad para evaluar mejor su correlación con la YFAS.

En resumen, la versión de YFAS en español utilizada en este estudio presentó propiedades psicométricas adecuadas y similares a las de la versión original y a las de otras traducciones y adaptaciones de la misma; por tanto, se considera que podría ser útil en la práctica asistencial y en estudios de investigación clínica en la población mexicana.

Contar con un instrumento como éste en español abre la posibilidad de detectar en pacientes mexicanos una conducta de hiperfagia que puede reflejar rasgos de trastornos psiquiátricos que lleven al desarrollo de la obesidad, lo que facilitaría su tratamiento, permitiendo un abordaje más amplio y acorde al fenómeno natural de la apetencia. De hecho, ampliará la perspectiva que se tiene de la obesidad agregando la posibilidad de equiparar las conductas adictivas que llevan a la ingesta excesiva con el modelo de trastornos por consumo de sustancias.

En cuanto a las limitaciones de este estudio se incluyen aquéllas que están en relación con el constructo de adicción a los alimentos, el cual está aún en discusión y por lo tanto no cuenta con criterios diagnósticos consensuados y validados, y mucho menos con otros instrumentos de medición 
con los cuales establecer una comparación. Por otra parte, la muestra es pequeña y perteneciente a un universo restringido, lo que hace que no sea representativa, disminuyendo así la validez externa de los resultados y el empleo de pruebas más robustas.

A pesar de las deficiencias mencionadas en el análisis estadístico empleado para este estudio, no se puede descalificar su utilidad e importancia y será el clínico quien decida su uso y trascendencia en vías de hacer otros más completos y sólidos estadísticamente.

\section{Financiamiento}

Esta investigación fue financiada por el CONACyT, número de proyecto 233918 de la convocatoria FOSISS 2014(PG).

\section{Conflicto de intereses}

Los autores declaran no tener ningún conflicto de intereses.

\section{Agradecimientos}

Le damos las gracias a la doctora Gearhardt por autorizarnos a llevar a cabo la validación en español de la YFAS en población mexicana. A la doctora María Isabel Sollozo por su asesoría con los análisis estadísticos. A las autoridades de la Escuela de Dietética y Nutrición del ISSSTE y al Centro de Dietética y Nutrición perteneciente a la Escuela, por permitirnos aplicar las escalas a los estudiantes y trabajadores de su institución.

\section{REFERENCIAS}

1. Siraj ES, Williams KJ. Another Agent for Obesity--Will This Time Be Different? N Engl J Med. 2015;373:82-83.

2. Novak NL, Brownell KD. Role of policy and government in the obesity epidemic. Circulation. 2012;126:2345-2352.

3. World Health Organization. Obesity and overweight. 2015. Disponible en: http://www.who.int/mediacentre/factsheets/fs311/en/ [Citado 9 de noviembre de 2016].

4. World Health Organization. Obesity. 2015. Disponible en: http:// www.who.int/topics/obesity/en/ [Citado 9 de noviembre de 2016].

5. Gale SM, Castracane VD, Mantzoros CS. Energy homeostasis, obesity and eating disorders: recent advances in endocrinology. J Nutr. 2004;134:295-298.

6. Sawchenko PE. Toward a new neurobiology of energy balance, appetite, and obesity: the anatomists weigh in. J Comp Neurol. 1998;402:435-441.

7. Spiegelman BM, Flier JS. Obesity and the regulation of energy balance. Cell. 2001;104:531-543.

8. Agras WS. Treatment of binge-eating disorder. In: Treatment of Psychiatric Disorders, edited by Gabbard GO. Washington DC: American Psychiatric Pubishing. 2001;2209-2219.

9. Devlin MJ, Yanovski SZ, Wilson GT. Obesity: what mental health professionals need to know. Am J Psychiatry. 2000;157:854-866.

10. Smith DE, Marcus MD, Lewis CE, Fitzgibbon M, Schreiner P. Prevalence of binge eating disorder, obesity, and depression in a biracial cohort of young adults. Ann Behav Med. 1998;20:227-232.

11. Wadden TA, Sarwer DB, Womble LG, Foster GD, McGuckin BG, Schimmel A. Psychosocial aspects of obesity and obesity surgery. Surg Clin North Am. 2001;81:1001-1024.

12. Williamson DA, Martin CK. Binge eating disorder: a review of the literature after publication of DSM-IV. Eat Weight Disord. 1999;4:103-114.

13. McElroy SL, Hudson JI, Capece JA, Beyers K, Fisher AC, Rosenthal
NR. Topiramate for the treatment of binge eating disorder associated with obesity: a placebo-controlled study. Biol Psychiatry. 2007;61:1039$1048,2007$.

14. Ramacciotti CE, Coli E, Bondi E, Burgalassi A, Massimetti G, Dell'osso L. Shared psychopathology in obese subjects with and without binge-eating disorder. Int J Eat Disord. 2008;41:643-649.

15. Specker S, de ZM, Raymond N, Mitchell J. Psychopathology in subgroups of obese women with and without binge eating disorder. Compr Psychiatry. 1994;35:185-190.

16. American Psychiatric Association. Diagnostic and statistical manual of mental disorders: DSM-IV. 4a ed. Arlington: American Psychiatric Publishing; 2000.

17. Britz B, Siegfried W, Ziegler A, Lamertz C, Herpertz-Dahlmann BM, Remschmidt H, Wittchen HU, Hebebrand J. Rates of psychiatric disorders in a clinical study group of adolescents with extreme obesity and in obese adolescents ascertained via a population based study. Int J Obes Relat Metab Disord. 2000; 24:1707-1714, 2000.

18. Bulik CM, Sullivan PF, Kendler KS. Medical and psychiatric morbidity in obese women with and without binge eating. Int J Eat Disord. 2002;32:72-78.

19. de Zwaan M. Binge eating disorder and obesity. Int J Obes Relat Metab Disord. 2001;25 Suppl 1:S51-S55.

20. Carter J.C, Davis C. An addiction model of binge eating disorder. In: Addiction medicine, Springer New York, 2011, p. 633-647.

21. Volkow ND, Wang GJ, Fowler JS, Tomasi D, Baler R. Food and drug reward: overlapping circuits in human obesity and addiction. Curr Top Behav Neurosci. 2012(11):1-24.

22. Volkow ND, Wang GJ, Tomasi D, Baler RD. Obesity and addiction: neurobiological overlaps. Obes Rev. 2013;14:2-18.

23. Tomasi D, Wang GJ, Wang R, Caparelli EC, Logan J and Volkow ND. Overlapping patterns of brain activation to food and cocaine cues in cocaine abusers: association to striatal D2/D3 receptors. Hum Brain Mapp. 2015;36:120-136.

24. Volkow ND, Wise RA. How can drug addiction help us understand obesity? Nat Neurosci. 2005;8:555-560.

25. American Psychiatric Association. Diagnostic and satistical manual of mental disorders: DSM-5. Washington, D.C. 2013.

26. Gearhardt AN, Corbin WR, Brownell KD. Preliminary validation of the Yale Food Addiction Scale. Appetite. 2009;52:430-436.

27. Bégin C, St-Louis ME, Turmel S, Tousignant B, Marion LP, Ferland F, Blanchette-Martin N, Gagnon-Girouard MP. Does food addiction distinguish a specific subgroup of overweight!obese overeating women?. Health. 2012;1492-1499.

28. Granero R, Hilker I, Aguera Z, Jimenez-Murcia S, Sauchelli S, Islam MA, Fagundo AB, Sanchez I, Riesco N, Dieguez C, Soriano J, Salcedo-Sanchez C, Casanueva FF, De la Torre R, Menchon JM, Gearhardt AN, Fernandez-Aranda F. Food addiction in a Spanish sample of eating disorders: DSM- 5 diagnostic subtype differentiation and validation data. Eur Eat Disord Rev. 2014;22: 389-396.

29. Meule A, Heckel D, Kubler A. Factor structure and item analysis of the Yale Food Addiction Scale in obese candidates for bariatric surgery. Eur Eat Disord Rev. 2012;20:419-422.

30. Meule A, Kubler A. Food cravings in food addiction: the distinct role of positive reinforcement. Eat Behav. 2012; 13:252-255.

31. Meule A, Vögele C, Kübler A. German translation and validation of the Yale Food Addiction Scale. Diagnostica. 2012;115-126.

32. Flint AJ, Gearhardt AN, Corbin WR, Brownell KD, Field AE, Rimm EB. Food-addiction scale measurement in 2 cohorts of middle-aged and older women. Am J Clin Nutr. 2014;99:578-586.

33. Gearhardt AN, Roberto CA, Seamans MJ, Corbin WR, Brownell KD. Preliminary validation of the Yale Food Addiction Scale for children. Eat Behav. 2013;14:508-512.

34. Grucza RA, Przybeck TR, Cloninger CR. Prevalence and correlates of binge eating disorder in a community sample. Compr Psychiatry. 2007;48:124-131. 
35. Gearhardt AN, Cobin WR, Brownell KD. Preliminary validation of the Yale Food Addiction Scale. Appetite. 2009;52:430-436.

36. Zúñiga $\mathrm{O}$, Robles R. Validez de constructo y consistencia interna del Cuestionario de Trastorno por Atracón en población mexicana con obesidad. Psiquis. 2006;15:126-134.

37. Gormally J, Black S, Daston S, Rardin D. The assessment of binge eating severity among obese persons. Addict Behav. 1982;7:47-55.

38. Marcus MD, Wing RR, Hopkins J: Obese binge eaters: Affect, cognition and response to behavioral weight control. J Consult Clin Psychol. 1988;3:433-439.

39. Timmerman GM: Binge Eating Scale: Further Assessment of Validity and Reliability. Journal of Applied Biobehavioural Research. 1999;4(1):1-12.

40. Freitas SR, Lopes CS, Appolinario JC, Coutinho W. The assessment of binge eating disorder in obese women: A comparison of the binge eating scale with the structured clinical interview for the DSM-IV. Eating Behaviours. 2006;7:282-228.

41. Ricca V, Mannucci E, Moretti S, Bernardo MD, Zucchi T, Cabras PL, Rotella CM: Screening for binge eating disorder in obese outpatients. Comprehensive Psychiatry. 2000; 41:111-115.
42. World Health Organization. BMI classification. 2015. Disponible en: http://apps.who.int/bmi/index.jsp?introPage=intro_3.html [Citado 9 de noviembre de 2016].

43. Cronbach LJ. Coefficient alpha and the internal structure of tests. Psychometrika. 1951;16:297-334.

44. Cortina J. What is coefficient alpha? An examination of theory and applications. Journal of Applied Psychology. 1993;78:98-104.

45. García-Mijares JF, Alejo-Galarza GJ, Mayorga-Colunga SR, Guerrero-Herrera LF, Ramírez-GarcíaLuna JL. Validación al español del SelfHarm Questionnaire para detección de autolesionismo en adolescentes. Salud Mental. 2015;38:287-292.

46. Marín-Navarrete R, Mejía-Cruz D, Templos-Núñez L, Rosendo-Robles A, González-Zavala M, Nanni-Alvarado R, García I, Leff P, Salazar-Juárez A and Antón-Palma B. Validation of a cocaine craving questionnaire (CCQ-G) in Mexican population. Salud Mental. 2011;491-496.

47. Tavakol M, Dennick R. Making sense of Cronbach's alpha. International Journal of Medical Education. 2011;53-55. 